\title{
Identification of key gene modules and transcription factors for human osteoarthritis by weighted gene co-expression network analysis
}

\author{
XIANG GAO, YU SUN and XU LI \\ Department of Orthopedic Surgery, The First Hospital of China Medical University, Shenyang, Liaoning 110000, P.R. China
}

Received October 2, 2018; Accepted July 8, 2019

DOI: $10.3892 /$ etm.2019.7848

\begin{abstract}
Osteoarthritis (OA) is one of the most prevalent causes of joint disease. However, the pathological mechanisms of OA have remained to be completely elucidated, and further investigation into the underlying mechanisms of OA development and the identification of novel therapeutic targets are urgently required. In the present study, the dataset GSE114007 was downloaded from the Gene Expression Omnibus database. Based on weighted gene co-expression network analysis (WGCNA) and the identification of differentially expressed genes (DEGs), the microarray data were further analyzed to identify hub genes, key transcription factors (TFs) and pivotal signaling pathways involved in the pathogenesis of OA. A total of 1,898 genes were identified to be differentially expressed between OA samples and normal samples. Based on WGCNA, the present study identified 5 hub modules closely associated with OA, and the potential key TFs for hub modules were further explored based on CisTargetX. The results demonstrated that B-Cell Lymphoma 6, Myelin Gene Expression Factor 2, Activating Transcription Factor 3, CCAAT Enhancer Binding Protein $\gamma$, Nuclear Factor Interleukin-3-Regulated, FOS Like Antigen-2, FOS-Like Antigen-1, Fos Proto-Oncogene, JunD Proto-Oncogene, Transcription Factor CP2 Like 1, RELA proto-oncogene NF-kB subunit, SRY-box transcription factor 3, V-Ets Avian Erythroblastosis Virus E26 Oncogene Homolog 2, Interferon Regulatory Factor 4 and REL proto-oncogene, NF-kB subunit were the potential key TFs. In addition, osteoclast differentiation, FoxO, MAPK and PI3K/Akt signaling pathways were revealed to be imperative for the pathogenesis of OA, as these 4 pivotal signaling pathways were observed to be tightly linked through 4 key TFs
\end{abstract}

Correspondence to: Professor Xu Li, Department of Orthopedic Surgery, The First Hospital of China Medical University, 155 Nanjing North Street, Shenyang, Liaoning 110000, P.R. China E-mail: likyokucmu@163.com

Key words: osteoarthritis, cartilage tissues, weighted gene co-expression network analysis, underlying pathogenesis, transcription factors
Fos Proto-Oncogene, JUN, JunD Proto-Oncogene and MYC, and 4 DEGs Vascular Endothelial Growth Factor A, Growth Arrest and DNA Damage Inducible $\alpha$, Growth Arrest and DNA Damage Inducible $\beta$ and Cyclin D1. The present study identified a set of potential key genes and signaling pathways, and provided an important opportunity to advance the current understanding of OA.

\section{Introduction}

Osteoarthritis (OA) is one of the most prevalent joint diseases, and has been predicted to be the greatest cause of disability among patients aged $>40$ years by 2040 (1). One study have revealed that the economic and humanistic burden of OA is high worldwide (2). A patient with OA typically presents with cartilage degeneration and synovial inflammation (3). Clinically, OA is characterized by pain, swelling, joint deformity and disability (4). In addition to operative treatment, the most common pharmacological therapies for OA are analgesic and non-steroidal anti-inflammatory drugs (5). Although a considerable amount of studies investigating OA have been published (6), a systematic understanding of the pathogenesis of OA and its potential therapeutic targets would be of great clinical significance.

With the development of high-throughput genomics technology, gene expression profiles obtained from microarrays have been extensively used to explore and examine genes associated with the progression of various diseases, including the pathogenesis of OA $(7,8)$. However, these studies may have been more relevant if they had considered the correlation of the expression between multiple genes, rather than just focused on the screening of differentially expressed genes (DEGs). Based on the idea that genes with similar expression patterns may be functionally associated, a novel biological methodology, weighted gene co-expression network analysis (WGCNA), has been developed (9). Based on WGCNA, complex microarray data may be simplified into several functional modules, which are composed of co-expressed genes. Subsequently, hub modules may be identified through the associations between modules and clinical traits. Due to the limited access to normal cartilage to serve as control groups, the majority of Bioinformatics studies are focused on knee synovial tissue. However, to the best of our knowledge, WGCNA has not been previously 
applied to analyze the cartilaginous expression profiles of OA.

The primary objective of the present study was to obtain data which will aid in providing a more detailed understanding of the pathogenesis of OA. The methodological approach taken in the present study is a mixed methodology based on WGCNA and the screening of DEGs. Microarray data with the accession number GSE114007 were downloaded in the present study, which were subjected to Bioinformatics analysis with the attempt to identify hub genes from a protein-protein interaction (PPI) network, and construct a co-expression network through WGCNA in order to identify hub modules associated with the pathogenesis of OA. Considering that the co-expressed genes in the same module may participate in the same biological function or signaling pathway, and from the point of view of co-expressed genes having the potential to be regulated by the same transcription factors (TFs), the present study attempted to identify key TFs involved in the pathogenesis of OA $(10,11)$.

\section{Materials and methods}

Data collection. The dataset GSE114007 [deposited by Fisch et al (11)], which is based on the Illumina HiSeq 2000 and Illumina HiSeq 500 platforms (Illumina, Inc.), was downloaded from the Gene Expression Omnibus (GEO) database (http://www.ncbi.nlm.nih.gov/geo/) (12). The GSE114007 dataset was selected for analysis as it contained the complete information comprising gene expression data and detailed clinical data in this database. RNA-sequencing (RNA-seq) was performed on 18 normal and 20 OA human knee cartilage tissues. Subsequently, the gene expression data were subjected to identical processing using the Robust Multichip Average function within the limma R package (version 3.24.15) (13) for background correction, $\log 2$ transformation, quantile normalization and median polish algorithm summarization. The microarray data probe was transformed to gene symbols with Bioconductor Annotation Data software packages (version 1.20.0) (14). In the case that 1 single gene symbol was captured by several probes, the final gene expression level was calculated from the average value of those probes. When one probe was mapped to multiple gene sets, information about the probe was deleted. A principal component analysis (PCA) of normalized data was performed in the present study using the prcomp algorithm in R software. PCA was used for unsupervised multivariate analyses in order to determine the directions of maximum covariance without referring to class labels (Normal/OA).

Identification of DEGs. The DEGs between OA and normal samples were identified through the limma $\mathrm{R}$ package using the Student's t-test (13). A $\mid \log _{2}$ (fold change) $\mid>1$ and adjusted $\mathrm{P}$-value of $<0.05$ were set as the cut-off criteria. To control the false discovery rate, adjusted P-values were computed for multiple testing corrections of the raw P-value through the Benjamini-Hochberg (BH) method (15). Volcano plots were also constructed to visually display the DEGs. DEGs were counted in the respective hub modules.
Weighted co-expression network construction. WGCNA is a widely used systems biology method, which may be used to construct a scale-free network from gene expression data. Based on the dataset GSE114007, which is comprised of 18 normal and 20 OA samples, a gene co-expression network was constructed through the WGCNA R package (version 1.68) (9). In order to determine whether there were any outlier samples, the present study performed sample clustering according to the distance between different samples in Pearson's correlation matrices. The correlation coefficient between genes ' $m$ ' and ' $n$ ' was defined as $\mathrm{s}_{\mathrm{m}, \mathrm{n}}=|\operatorname{cor}(\mathrm{m}, \mathrm{n})|$, with the gene expression correlation matrix being $S=\left[\mathrm{s}_{\mathrm{mn}}\right]$. The correlation matrix was subsequently translated into an adjacency matrix, $\mathrm{A}=\left[\mathrm{a}_{\mathrm{mn}}\right]$, through a power function $\mathrm{a}_{\mathrm{mn}}=\mathrm{s}_{\mathrm{mn}}{ }^{\beta}$. $\beta$ was defined as the soft threshold parameter. The $\beta$-value was selected as long as scale-free topology fit index $\left(\mathrm{R}^{2}\right)$ reached 0.9 . The adjacency matrix was then transformed into a topological overlap matrix (TOM) through the following algorithm:

$$
\varpi_{m n}=\frac{l_{m n}+\alpha_{m n}}{k_{m} k_{n}+1-\alpha_{m n}}
$$

$l_{\mathrm{mn}}$ indicates the product sums of the adjacency coefficients of the nodes connected to both $\mathrm{m}$ and $\mathrm{n} . \alpha_{m n}$ indicates the correlation coefficient between $\mathrm{m}$ and $\mathrm{n} . k_{m}$ indicates the sum of the adjacency coefficients of the nodes only connected to m. $k_{n}$ indicates the sum of the adjacency coefficients of the nodes only connected to $\mathrm{n}$. Co-expressed gene modules were identified by the dynamic tree cut method, which performs adaptive branch pruning based on various criteria, including a minimum cluster size of 30 genes and a cut height of 0.25 . Modules with highly correlated genes were detected and genes exhibiting weaker connectivity were left unassigned. Hierarchical clustering was used to produce a dendrogram of genes based on the corresponding dissimilarity of TOM, and to classify genes with similar expression patterns into gene modules. The module eigengene (ME) was calculated using the MEs function in the 'WGCNA' R package. Finally, hub modules were detected based on the correlation between ME and clinical traits. The correlation values $>0.6$ were set as cutoff criteria.

PPI network construction and identification of hub genes. The Search Tool for the Retrieval of Interacting Genes (STRING) database (http://www.string-db.org/; version 11.0) was used for the construction of the protein-protein interaction (PPI) network for DEGs and hub modules using default parameters in the STRING database. The types of connection included the following items: Textmining, Experiments, Databases, Co-expression, Neighborhood, Gene fusion and Co-occurrence. To extract valid interactions, a confidence score of $>0.4$ was set as the cut-off criterion. The nodes in the PPI network with the highest degrees of interactions were considered as hub proteins. The present study also used the Cytoscape plugin CytoHubba to calculate the degree of each node in the PPI network (version 1.5) (16). The top 10 genes in each module and top 10 DEGs were defined as hub genes. In addition, OA-associated genes were downloaded from Junior Doc (http://www.drwang.top/). By entering 'OA' in the search tool, 520 OA-associated genes were listed and downloaded. 
Finally, OA-associated genes were counted in the respective hub modules.

Functional enrichment analysis of DEGs and hub modules. To determine the DEGs and hub modules involved in BP terms and pathways, Gene ontology (GO) and Kyoto Encyclopedia of Genes and Genomes (KEGG) pathway enrichment analyses were performed using the ClusterProfiler $\mathrm{R}$ package (version 3.8.1) (17). An adjusted P-value $<0.01$ (to control the false discovery rate, adjusted $\mathrm{P}$-values were computed for multiple testing corrections of the raw $\mathrm{P}$-value through the $\mathrm{BH}$ method) was set as the cutoff criterion for the functional enrichment analysis.

Identification of key TFs. CisTargetX may be used to predict TFs involved in the regulation of co-expressed genes $(18,19)$. Based on CisTargetX, the present study further explored the potential TFs for each hub module. CisTargetX was used with the following parameters: Mapping of genes in regions, ' $10 \mathrm{~kb}$ upstream, transcription start site, $10 \mathrm{~kb}$ downstream'; minimum fraction of overlap, ' 0.4 '; normalized enrichment score threshold, '4'; receiver operating characteristic threshold for area under the curve calculation, '0.005'. Key TFs of each hub module were defined as predicted TFs, which were also DEGs in the present study. Common elements in DEGs and predicted TFs were compared using Microsoft Excel (version 14.5.1; Microsoft Corporation). By comparing the genes targeted by key TFs with DEGs, the DEGs that were targeted by the key TFs were counted and extracted for further study.

Identification of pivotal signaling pathways. To provide a deeper understanding of changes in gene regulation underlying OA, information on key TFs likely involved in regulating these DEGs was extracted. KEGG analysis was subsequently performed for key TFs and their target DEGs in order to identify pivotal signaling pathways. Based on STRING database and the pheatmap R package (https://cran.r-project. org/web/packages/pheatmap/index.html; version 1.0.12), a PPI network (confidence score $>0.4$ set as the cut-off criterion) and heatmap were constructed for key TFs and their target DEGs that were involved in key signaling pathways. The transcriptional regulatory network was visualized by Cytoscape.

\section{Results}

Identification of DEGs. To estimate false-positives, the procedure was performed using randomized versions of the sample labels and the outcomes were compared with the original results based on PCA (Fig. 1A) (20). PCA revealed clear differences between the normal and OA samples. Sample clustering revealed that no samples required removal from subsequent analysis due to outliers (Fig. 1B). With an adjusted P-value $<0.05$ and $\mid \log 2$ fold changel $>1$ as the cut-off criteria, a total of 1,898 genes were identified to be differentially expressed between OA samples and normal samples, of which 966 were upregulated (Table SI) and 932 were downregulated (Table SII). The DEGs are highlighted in the volcano plot (Fig. S1). As illustrated in Fig. S2, unsupervised hierarchical clustering analysis of the obtained DEGs was able to distinctly classify all samples into normal and OA groups.

Weighted co-expression network construction. A weighted co-expression network was constructed using the 'WGCNA' $\mathrm{R}$ package. Construction of an appropriate scale-free network required an optimal soft-thresholding power to which co-expression similarity was raised to calculate adjacency. The analysis of network topology was performed for various soft-thresholding powers in order to obtain a relatively balanced scale independence and mean connectivity of the WGCNA (9). In Fig. 2A, the scale-free topology fitting index $\left(\mathrm{R}^{2}, \mathrm{y}\right.$-axis) is presented as a function of the soft-thresholding power (x-axis) in the left panel, while the right panel displays the mean connectivity (degree, $y$-axis) as a function of the soft-thresholding power (x-axis). Red Arabic numbers in the panels denote different soft-thresholds. The red line in the left panel indicates $R^{2}=0.9$. There was a trade-off between maximization of the scale-free topology model fitting index $\left(\mathrm{R}^{2}\right)$ and maintenance of a high mean number of connections. Thus, $\beta$ was set as 9 . Subsequently, co-expressed gene modules were identified by the dynamic tree cut method, and each of the modules was marked by a color, indicated in the color band below the dendrogram (Fig. 2B). Genes that were not assigned to any of the modules were colored in grey. A total of 24 modules were identified in the GSE114007 dataset (Fig. 3), of which 5 modules were identified as hub modules based on the correlation coefficients between the ME and traits $(>0.6)$. The genes in each module are listed in Table SIII. The purple, salmon, black, brown and magenta modules were close to 'OA' traits. Among them, the purple, black and brown modules were also close to 'age' traits. Previous studies have concluded that females are more susceptible to OA than males (21). However, the present study did not identify any modules that were closely associated with 'sex' traits. Detailed information of hub modules is listed in Table I.

GO enrichment function and pathway analysis. To further investigate the DEGs and hub modules involved in BP terms and signaling pathways, respective GO and KEGG analyses were performed using the clusterProfiler R package (Fig. 4). The results demonstrated that the DEGs were significantly enriched in key processes, including skeletal system development, ossification, extracellular structure organization, osteoblast differentiation, response to corticosteroid, focal adhesion, the Wnt signaling pathway, complement and coagulation cascades, extracellular matrix (ECM)-receptor interactions and rheumatoid arthritis (Fig. 4A). Fig. 4B-G presents the results obtained from the enrichment analysis of hub modules, which suggest that these BP terms and pathways may exert pivotal functions in the pathogenesis of OA.

PPI network analysis of DEGs and hub modules. A PPI network for the DEGs at the protein level was constructed using the STRING analysis tool. A total of 537 nodes and 1,218 edges were contained in the PPI network. Based on the plugin CytoHubba, the hub genes were defined as the genes with the top 10 with the highest degree of connectivity in the entire PPI network (Table II), including Jun Proto-Oncogene (JUN), Vascular Endothelial Growth Factor A (VEGFA), Fos 
A

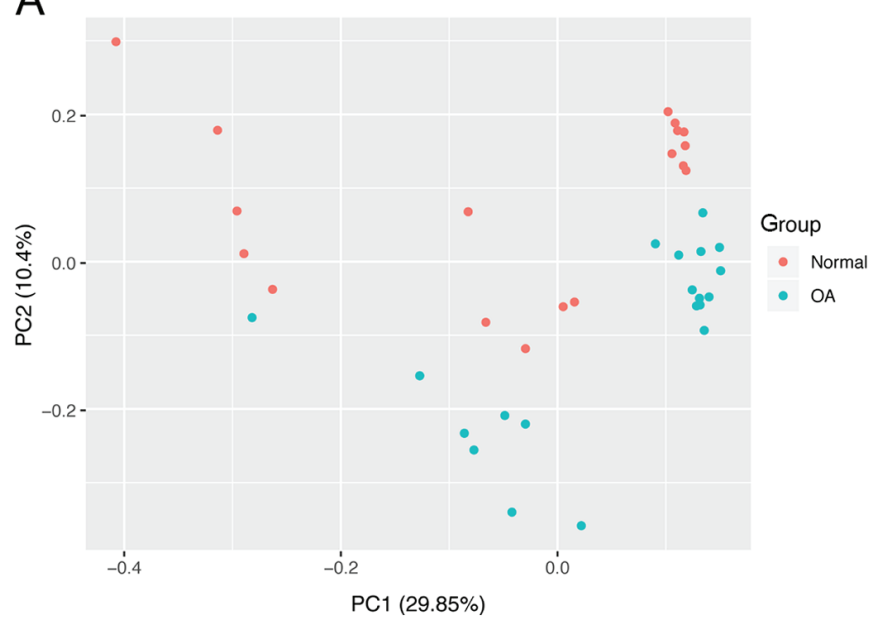

B

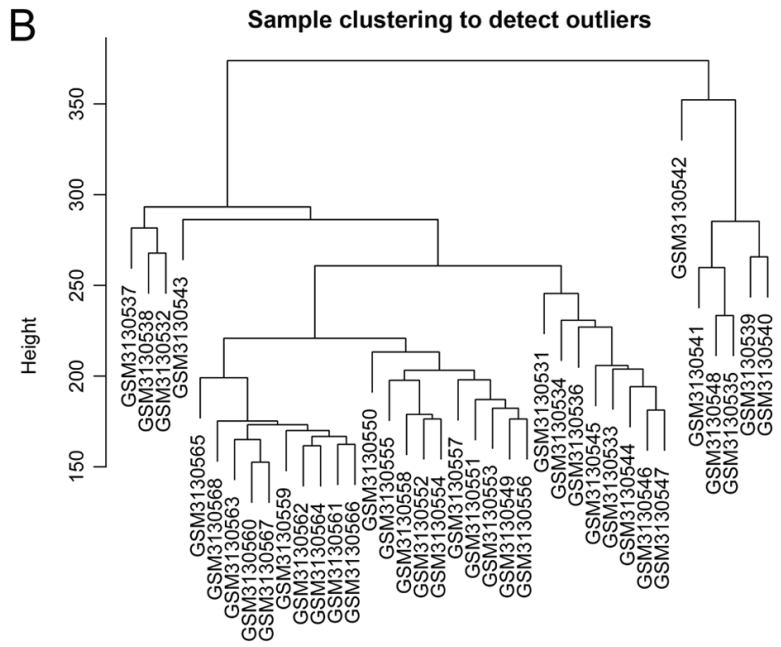

Figure 1. PCA and sample clustering. (A) PCA based on PC1 (proportion of contribution, 59.3\%) and of PC2 (proportion of contribution, 23.1\%) indicated a different distribution of normal and osteoarthritis samples. (B) Sample clustering indicated that no outliers were present that required removal from the subsequent analysis. FC, fold change; PCA, principal component analysis.
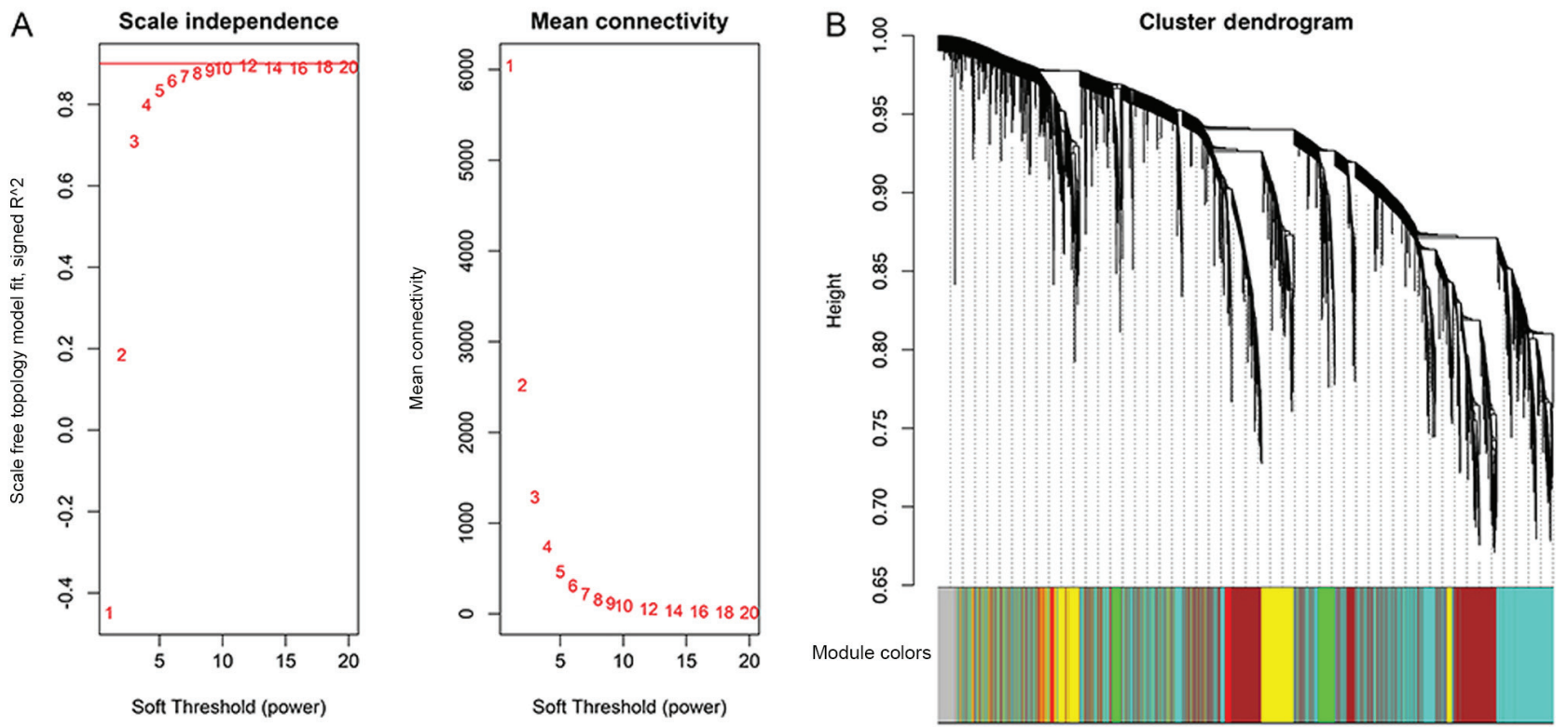

Figure 2. Weighted gene co-expression network construction. (A) Analysis of the scale free topology module fit and the mean connectivity for different soft-thresholding powers $(\beta)$. The red line in the left panel indicates $\mathrm{R}^{2}=0.9$. (B) Gene dendrogram obtained from hierarchical clustering. The row of colored bars below the dendrogram represents the module assignment identified by the dynamic tree cut.

Proto-Oncogene (FOS), DNA Topoisomerase II $\alpha$ and Matrix Metallopeptidase 9. A PPI network of hub modules was constructed using the same method, and genes with the top 10 connectivity in the hub modules were defined as the hub genes (Table II), including Cell Division Cycle 42 (CDC42), PH Domain and Leucine Rich Repeat Protein Phosphatase 1 (PHLPP1), Protein Tyrosine Phosphatase Receptor Type C (PTPRC), JUN and Ribosome Production Factor 2 (RPF2).

Identification of key TFs for hub modules. Transcriptional regulation is a fundamental and pivotal process involved in the pathogenesis of various diseases (22). Co-expressed genes in each hub module may be involved in the same biological functions and regulated by the same TFs. Therefore, the present study further explored the potential
TFs for hub modules based on CisTargetX (Table SIV). The results demonstrated that B-Cell Lymphoma 6 (BCL6), Myelin Gene Expression Factor 2 (MYEF2), Activating Transcription Factor 3 (ATF3), CCAAT Enhancer Binding Protein $\gamma$ (CEBPG), Nuclear Factor Interleukin-3-Regulated (NFIL3), FOS Like Antigen-2 (FOSL2), FOS-Like Antigen-1 (FOSL1), Fos Proto-Oncogene (FOS), JunD Proto-Oncogene (JUND), Transcription Factor CP2 Like 1 (TFCP2L1), RELA proto-oncogene NF-kB subunit (RELA), SRY-box transcription factor 3, V-Ets Avian Erythroblastosis Virus E26 Oncogene Homolog 2, Interferon Regulatory Factor 4 (IRF4) and REL proto-oncogene NF-kB subunit (REL) were the potential regulators involved in managing hub modules (Table I). Detailed information of key TFs is listed in Table SV. These TFs may serve a key role in regulating the 


\begin{tabular}{|c|c|c|c|c|}
\hline \multirow[b]{2}{*}{ MElightcyan } & \multicolumn{3}{|c|}{ Module-trait association } & \\
\hline & $-0.21(0.2)$ & $-0.2(0.2)$ & $-0.18(0.3)$ & \\
\hline MEpurple & $-0.94(1 e-18)$ & $-0.32(0.05)$ & $-0.72(4 e-07)$ & $T^{1}$ \\
\hline MEsalmon & $-0.67(4 \mathrm{e}-06)$ & $-0.19(0.3)$ & $-0.48(0.002)$ & \\
\hline MEdarkred & $-0.2(0.2)$ & $0.14(0.4)$ & $-0.18(0.3)$ & \\
\hline MEgreen & $-0.32(0.05)$ & $-0.17(0.3)$ & $-0.16(0.3)$ & \\
\hline MEyellow & $-0.045(0.8)$ & $-0.14(0.4)$ & $0.022(0.9)$ & \\
\hline MEmidnightblue & $0.19(0.2)$ & $-0.18(0.3)$ & $0.19(0.2)$ & -0.5 \\
\hline MEblack & $0.71(7 e-07)$ & $0.13(0.4)$ & $0.61(5 e-05)$ & \\
\hline MEbrown & $0.79(3 e-09)$ & $0.21(0.2)$ & $0.62(3 e-05)$ & \\
\hline MEmagenta & $0.64(2 \mathrm{e}-05)$ & $0.2(0.2)$ & $0.47(0.003)$ & \\
\hline MElightyellow & $0.22(0.2)$ & $-0.018(0.9)$ & $0.26(0.1)$ & \\
\hline MEroyalblue & $0.19(0.3)$ & $0.2(0.2)$ & $0.19(0.2)$ & \\
\hline MEtan & $0.17(0.3)$ & $-0.16(0.3)$ & $0.2(0.2)$ & -0 \\
\hline MEblue & $0.21(0.2)$ & $0.013(0.9)$ & $0.26(0.1)$ & \\
\hline MEcyan & $-0.19(0.3)$ & $-0.15(0.4)$ & $-0.17(0.3)$ & \\
\hline MEpink & $0.0076(1)$ & $-0.21(0.2)$ & $0.048(0.8)$ & \\
\hline MEgrey60 & $0.33(0.04)$ & $0.26(0.1)$ & $0.24(0.2)$ & \\
\hline MEturquoise & $-0.087(0.6)$ & $0.034(0.8)$ & $-0.18(0.3)$ & -0.5 \\
\hline MElightgreen & $-0.23(0.2)$ & $-0.15(0.4)$ & $-0.18(0.3)$ & \\
\hline MEdarkturquoise & $0.36(0.03)$ & $-0.011(0.9)$ & $0.16(0.3)$ & \\
\hline MEdarkgreen & $0.29(0.07)$ & $-0.085(0.6)$ & $0.23(0.2)$ & \\
\hline MEgreenyellow & $-0.31(0.06)$ & $-0.21(0.2)$ & $-0.29(0.07)$ & \\
\hline MEred & $-0.27(0.1)$ & $-0.15(0.4)$ & $-0.28(0.09)$ & $L_{-1}$ \\
\hline MEgrey & $0.12(0.5)$ & $0.67(5 e-06)$ & $0.019(0.9)$ & \\
\hline
\end{tabular}

Figure 3. Module-trait matrixes were identified based on the correlation between clinical traits and ME. The number on the left of each cell illustrates the corresponding correlation coefficient and the number in bracket on the right is the P-value. High correlation coefficients were colored in red and low correlation coefficients in blue. The cutoff value for age was set as 60 years. ME, module eigengene; OA, osteoarthritis.

network of hub modules, and potentially affect the initiation and progression of $\mathrm{OA}$.

Identification of pivotal signaling pathways. The key TFs that target the largest number of DEGs were in the purple, brown and magenta modules (Fig. 5A), and were therefore further investigated. Fig. 5B provides a heat map on 19 key TFs. KEGG analysis was subsequently performed for key TFs and their target DEGs in the purple, brown and magenta modules (Fig. 5C). Based on the results of this enrichment analysis, it was indicated that osteoclast differentiation (purple and magenta modules), forkhead box (Fox)O signaling pathway (brown and purple modules), mitogen-activated protein kinase (MAPK) signaling pathway (purple module) and PI3K/Akt signaling pathway (brown module) are imperative for the pathogenesis of OA. Based on analysis using the STRING database and the pheatmap $\mathrm{R}$ package, a PPI network and heatmap were respectively constructed for key TFs and their target DEGs that were involved in the pivotal signaling pathways (Fig. 6). This analysis highlights that these key TFs and DEGs serve a central role in the pivotal signaling pathways. It was also observed that the 4 pivotal signaling pathways are tightly linked through 4 key TFs, including FOS, JUN, JUND and MYC, as well as 4 DEGs, including VEGFA, GADD45A, GADD45B and CCND1. In addition to these 8 genes, the present study also revealed that BCL6 is the key TF that targets the largest number of DEGs, and that BCL6 is also involved in the FoxO signaling pathway. These results suggest that FOS, JUN, JUND, MYC, VEGFA, CCND1, GADD45A, GADD45B and BCL6 serve important roles in the pathogenesis of OA.

\section{Discussion}

OA is a common chronic joint disease among aging individuals, and is characterized by articular cartilage degeneration, thickening of synovial lining, subchondral bone sclerosis and the formation of osteophytes at the joint margin (23). However, there is currently no efficient treatment for OA. Therefore, it is imperative to understand the underlying molecular mechanisms and pathological processes governing OA. Due to the limited access to normal cartilage to serve as a control group, microarray data in the GEO database containing normal cartilage tissues are relatively sparse, and the sample size is frequently insufficient. For the present study, a total of 64 articles were screened out from the Pubmed database based on the following search terms applied to title and abstract only: 'Osteoarthritis' AND ('co-expression' OR 'coexpression' OR 'networks') AND gene expression. The abstract of these 64 studies was carefully read and these articles are summarized in Table SVI. As indicated in this table, the samples in most of 
A
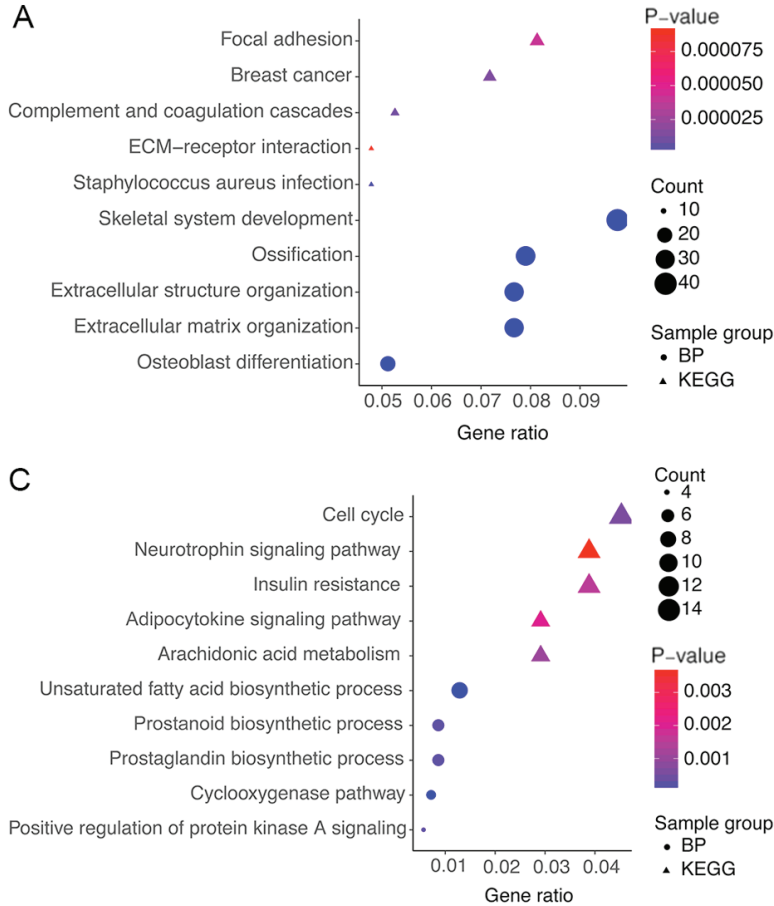

E

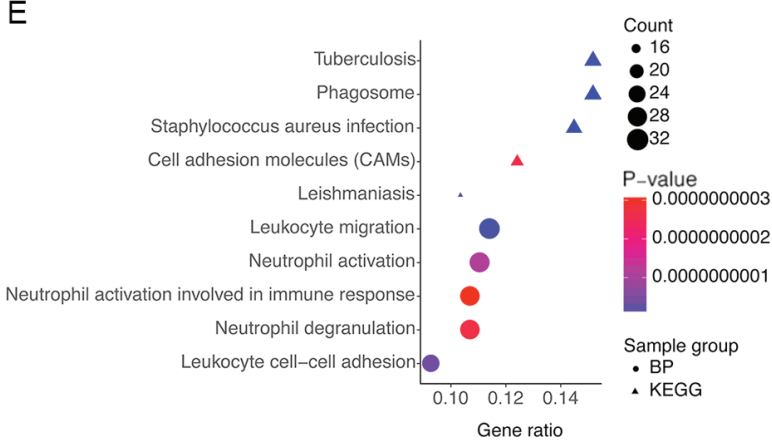

B

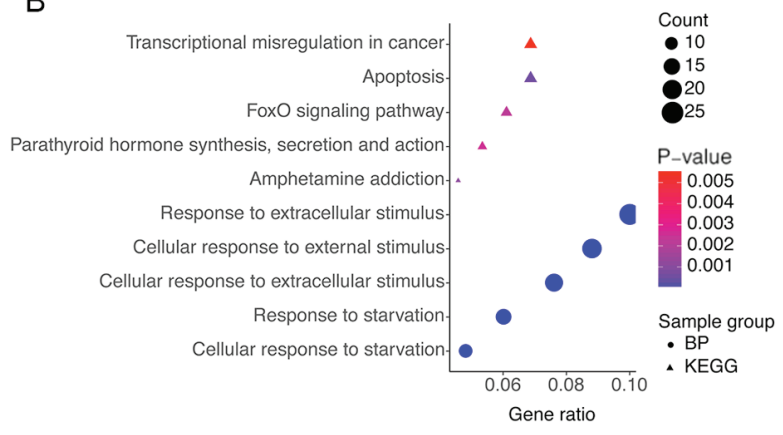

$D$

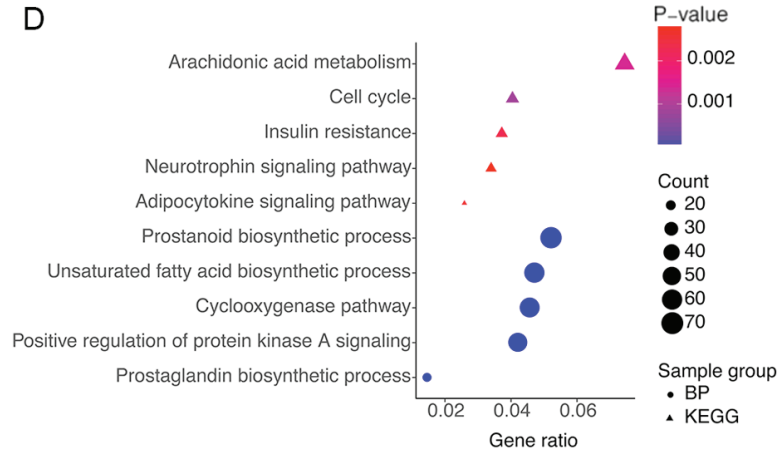

$\mathrm{F}$

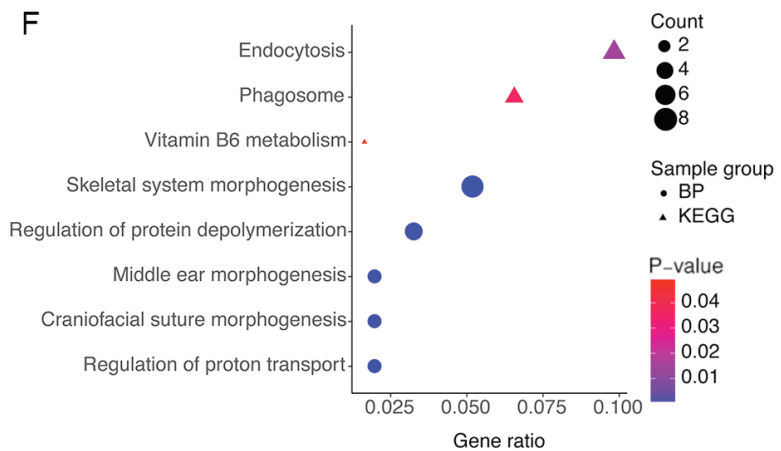

Figure 4. Functional enrichment analysis for DEGs and hub modules. (A) Enrichment analysis for all DEGs. (B-F) Enrichment analysis for individual modules. (B) Purple, (C) black, (D) brown, (E) magenta and (F) salmon module. Triangles represent GO terms and circles represent KEGG pathways. DEG, differentially expressed gene; KEGG, Kyoto Encyclopedia of Genes and Genomes; BP, biological process; ECM, extracellular matrix; Fox, forkhead box.

the studies are from knee synovial tissue, while three studies investigated the microarray data containing samples of human knee articular cartilage $(11,24,25)$. The GSE114007 dataset contained 18 normal and 20 OA human knee cartilage tissues. The cartilage tissues were obtained from the weight-bearing regions on medial and lateral femoral condyles. The samples for the normal group were collected from joints that had no macroscopic or microscopic evidence of cartilage damage, so that the differences between normal and OA samples are obvious. Another two datasets in the GEO database are worth mentioning, namely GSE117999 and GSE113825, which contain data on human knee cartilage tissues. Degenerative OA tends to be initiated in weight-bearing regions. Therefore, the major drawback of GSE117999 is that the healthy-appearing cartilage was garnered from the non-weight-bearing site of the knee joint. The dataset GSE113825 is restricted by the amount of samples. On the basis of the above, it was we concluded that GSE114007 is a valuable microarray dataset, and is worthy of further in-depth analysis based on WGCNA. To the best of our knowledge, the WGCNA has not been previously applied to analyze the expression profiles of cartilaginous OA. The results of the present study revealed that there were a total of 1,898 DEGs, including 966 upregulated and 932 downregulated DEGs in human knee cartilage tissues of OA. In addition, 5 hub modules were identified to be closely associated with OA based on WGCNA. A PPI network was constructed in order to identify hub genes among DEGs and hub modules. Functional enrichment analysis was also performed for the DEGs and hub modules in order to identify significant BP terms and signaling pathways. It is well-accepted that co-expressed genes are more likely to be co-regulated (26), and transcriptional regulation is a basic and critical BP in eukaryotes (22). Therefore, the further analysis focused on TFs, and 19 potential key TFs were identified, which may be involved in the regulation of hub modules based on CisTargetX. Finally, 4 pivotal signaling pathways that may largely contribute to the molecular processes of OA were identified via KEGG enrichment analysis. These signaling pathways are i) Osteoclast differentiation; ii) FoxO signaling pathway; iii) the MAPK signaling pathway, and iv) the PI3K/Akt signaling pathway.

Based on the WGCNA performed in the present study, 5 hub modules with highly relevant expression patterns that 
Table I. Detailed information of hub modules.

\begin{tabular}{llccccl}
\hline $\begin{array}{l}\text { Module } \\
\text { name }\end{array}$ & Trait & $\begin{array}{c}\text { Gene } \\
\text { count }\end{array}$ & $\begin{array}{c}\text { Module association } \\
\text { to trait }\end{array}$ & $\begin{array}{c}\text { OA associated } \\
\text { genes count }\end{array}$ & $\begin{array}{c}\text { DEG } \\
\text { count }\end{array}$ & \multicolumn{1}{c}{ Key TFs } \\
\hline Brown & OA/Age & 1,741 & $0.79 / 0.62$ & 77 & 594 & BCL6, MYEF2 \\
Purple & OA/Age & 307 & $-0.94 /-0.72$ & 14 & 180 & JUN, ATF3, CEBPG, NFIL3, FOSL2, \\
& & & & & 4 & FOSL1, FOS, MYC, JUND \\
Salmon & OA & 202 & -0.67 & 22 & 316 & ETS2, SOX9, SOX10 \\
Black & OA/Age & 920 & $0.71 / 0.61$ & 18 & 159 & IRF4, REL \\
Magenta & OA & 328 & 0.64 & 57 T & \\
\hline
\end{tabular}

OA, osteoarthritis; TFs, transcription factors; DEG, differentially expressed gene.

Table II. Top 10 hub genes in protein-protein interaction network of DEGs and hub modules.

\begin{tabular}{ll}
\hline DEGs/Modules & Top 10 hub genes \\
\hline DEGs & JUN VEGFA FOS TOP2A MMP9 CDK1 BIRC5 PTPRC KIT KIF23 \\
Black & CDC42 CDK2 ACTC1 EHHADH CDK5 INSR DLG4 ACSL4 ACE E2F4 \\
Brown & PHLPP1 EGF MYC CALM1 CCND1 CDC20 IGF1 AURKA NOTCH1 APP \\
Magenta & PTPRC CDK1 KIF15 TOP2A BUB1 CSF1R TYROBP ITGAM MELK BUB1B \\
Purple & JUN FOS VEGFA ATF3 EGR1 CDKN1A DUSP1 YARS JUNB FOSB \\
Salmon & RPF2 NGDN RCL1 RSL24D1 AEN TRMT11 ZNRD1 PAN2 SRSF7 DDX39A \\
\hline
\end{tabular}

DEG, differentially expressed gene.

were closely associated with OA were identified. GO BP and KEGG analyses were subsequently performed to identify significant BP terms and pathways in each hub module. The genes in the black module were primarily enriched in monocarboxylic acid biosynthetic process, signal transduction by p53 class mediator, cell cycle, insulin signaling pathway and FoxO signaling pathway. Monocarboxylate transporter 4 is a potential therapeutic target for inflammatory arthritis (27) and Zhu et al (28) reported that p53 genes are correlated with the disease grade of knee OA. The brown module was primarily enriched in skeletal system development, extracellular structure organization, ECM organization, ossification, chondrocyte differentiation, collagen catabolic process, PI3K/Akt signaling pathway, Hippo signaling pathway and FoxO signaling pathway. It has previously been reported that the chondrocyte ECM serves an important role in cartilage function (29), and that the dynamic equilibrium between matrix synthesis and degradation maintains the stability and integrity of cartilage tissue (30). A recent study has reported that the Hippo signaling pathway and ECM-receptor pathway are involved in the process of osteoarthritic chondrocyte apoptosis (31). In addition, the results of the present study revealed that the purple module was primarily enriched in response to extracellular stimulus, FoxO signaling pathway and human T-cell leukemia virus 1 infection. Andriacchi et al (32) reported that a mechanical stimulus at the baseline may enhance the sensitivity of a biomarker to predict cartilage thinning in a 5-year follow-up in patients with knee OA through a systems model of OA. In addition, the magenta module was typically enriched in neutrophil-mediated immunity and neutrophil activation involved in immune response. The magenta module is principally associated with the immune response. A previous study has suggested that the innate immune response is a potentially modifiable process, which may subsequently augment the pathological changes observed in OA (33).

The pathogenesis of OA is multifactorial and intricate, and is influenced by inflammation and oxidative stress (34). Due to its complex mechanism, numerous genes are differentially expressed in OA. In order to efficiently identify the crucial candidate genes, the present study primarily focused on the TFs likely involved in the pathogenesis of OA. A holistic approach was utilized, in which hub genes, key TFs and pivotal signaling pathways were integrated to identify more effective targets, which may be assessed in subsequent research. This analysis identified 8 key TFs and 1 hub gene (JUN, JUND, FOS, FOSL1, FOSL2, MYC, BCL6, ATF3 and VEGFA) that exert major functions in regulating hub modules and pivotal signaling pathways. Among these genes, JUN, FOS, MYC, ATF3 and VEGFA are also the hub genes in the PPI network of DEGs and the hub modules (Table II). JUN, JUND, FOS, FOSL1 and FOSL2 are subunits of activator protein 1 (AP-1) (35). Previous studies have suggested that AP-1 is involved in the differentiation, hypertrophy and expression of matrix metalloproteinase (MMP)13 in chondrocytes (36-38). In addition, Papachristou et al (39) identified that AP-1 is involved in the signal transduction pathway of mechanical loading in condylar chondrocytes. The results of the present study indicate that JUN, JUND, FOS, FOSL1 and FOSL2 are 


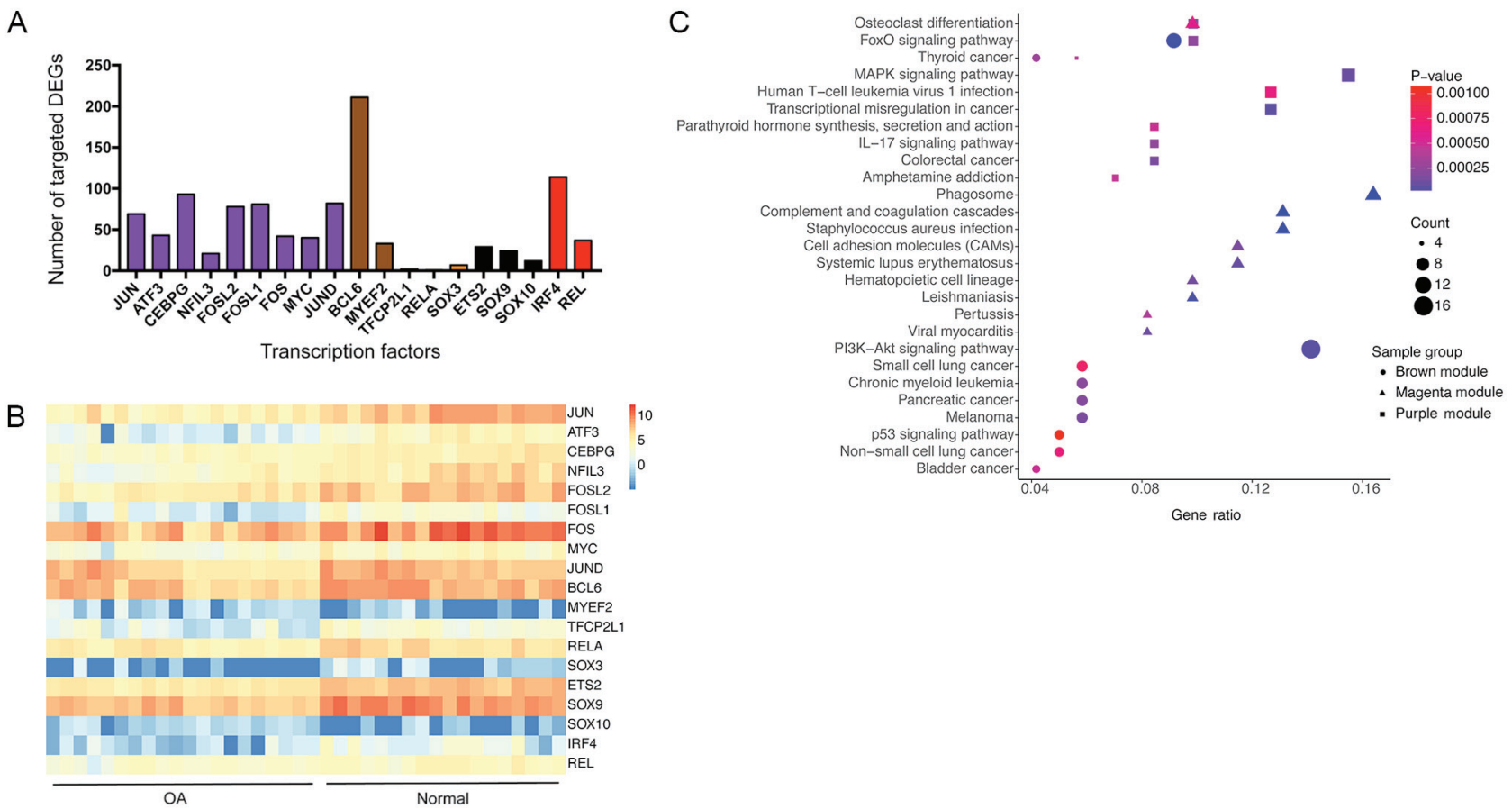

Figure 5. Identification of key TFs for hub modules. (A) Number of DEGs targeted by predicted TFss in each hub module. (B) Heatmap of predicted TFs based on the GSE114007 dataset between OA and normal samples. (C) Kyoto Encyclopedia of Genes and Genomes analysis for key TFs and their target DEGs. DEG, differentially expressed gene; OA, osteoarthritis; Fox, forkhead box; IL, interleukin; MAPK, mitogen-activated protein kinase; TF, transcription factor.

involved in osteoclast differentiation. In addition, previous research has suggested that AP-1 proteins are important inflammatory regulators in skin disease (40) and are hypothesized to be involved in regulating reactive oxygen species (ROS) in macrophages (41). There is evidence to suggest that C-myc suppresses apoptosis and promotes the proliferation of chondrocytes to prevent the occurrence and subsequent progression of OA via inactivating the MAPK signaling pathway (42). Consistent with the literature, the present study identified that MYC participates in the MAPK signaling pathway and is downregulated in OA samples. Lemos et al (43) demonstrated that inhibition of MYC in vivo as well as in human kidney organoids in vitro abrogated inflammation and fibrosis. The results of Anderton et al (44) suggest that MYC may serve a role in regulating a major anti-oxidant pathway downstream of glutamine. The present study identified BCL6 as the key TF that targeted the largest number of DEGs in the brown module. The precise association between OA and BCL6 has remained to be determined. However, a previous study reported that BCL6 attenuates lipopolysaccharide-induced inflammation in HK-2 cells via negative regulation of NLRP3 transcription (45). Iezaki et al (46) argued that ATF3 modulates the expression of MMP13 in human chondrocytes and indicated that ATF3 is implicated in the pathogenesis of OA through the modulation of inflammatory cytokine expression in chondrocytes, which may serve as a novel therapeutic target for the treatment of OA. ATF3 has been reported to be involved in regulating the inflammatory process in renal ischemia-reperfusion injury (47) and the central nervous system (48). With increasing severity of OA, greater vascular invasion into articular cartilage has been observed (49), and VEGFA has a fundamental role in angiogenesis (50). The present study observed that VEGFA is an integral DEG and in the center of a regulatory network
(Fig. 6). CCND1 is an important cell cycle regulator, and CCND1 silencing may promote interleukin (IL)-1 $\beta$-induced apoptosis in rat chondrocytes (51). SOX9, a TF known to regulate type II collagen and aggrecan, is a critical factor in the OA-associated downregulation of ECM molecules (52). According to a recent Bioinformatics study, VEGFA, JUN, ATF3, JUN and MYC were also significantly dysregulated in synovial membranes between the normal and OA group (53). These results suggest that dysregulation of these TFs and hub DEGs may participate in chondrocyte homeostasis and the pathogenesis of OA. Fisch et al (11) systematically analyzed the dataset GSE114007 based on a set of methods (identification of DEGs, TF enrichment analysis and network analysis), and obtained numerous significant results. They identified eight TFs (JUN, EGR1, JUND, FOSL2, MYC, KLF4, RELA and FOS) and 3 pivotal signaling pathways (hypoxia-inducible factor-1 signaling, pathways in cancer and FoxO signaling) that have a central role in the pathogenesis of OA. The present study used a different method, WGCNA, to further analyze the dataset GSE114007. The present analysis confirmed several of the key TFs identified by Fisch et al (11), including JUN, JUND, FOS, FOSL1, FOSL2, MYC and RELA, and suggested further key genes that may be involved in the pathogenesis of OA, including ATF3, BCL6, SOX9, VEGFA, CCND1, GADD45A and GADD45B. In addition to FoxO signaling, 3 further pivotal signaling pathways were identified (osteoclast differentiation, MAPK and PI3K/Akt signaling pathway).

In addition to the genes that have already been discussed, there are still other key TFs that target large numbers of DEGs and hub genes with high connectivity in PPI networks identified at present study that require further investigation, including GADD45A, GADD45B, CDC42, CEBPG, NFIL3, MYEF2, IRF4, REL, PHLPP1, 
A

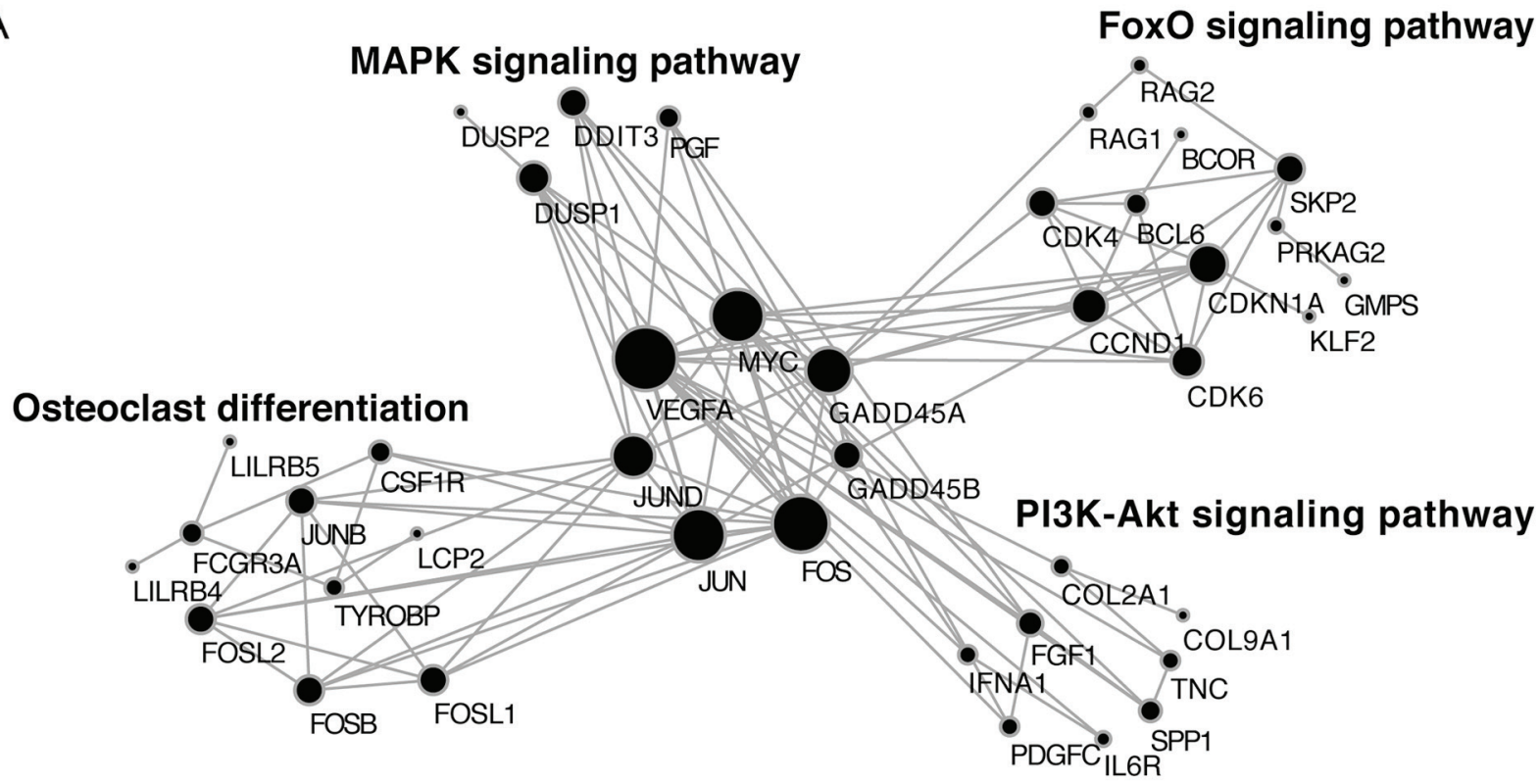

B

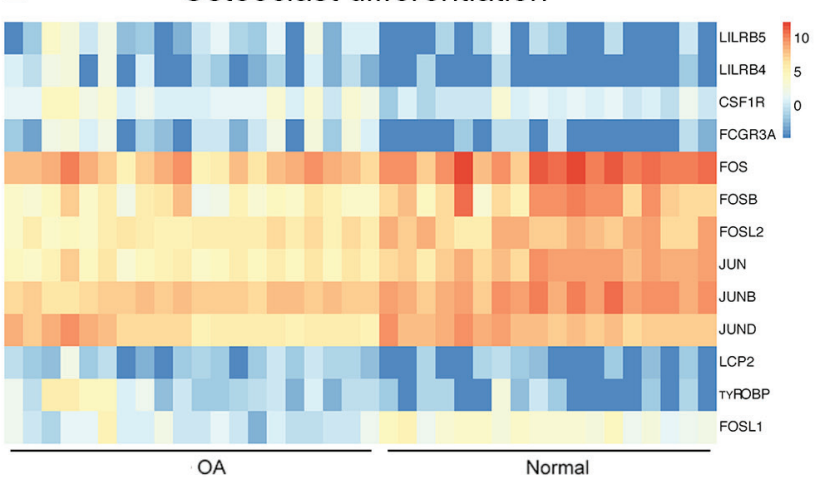

MAPK signaling pathway

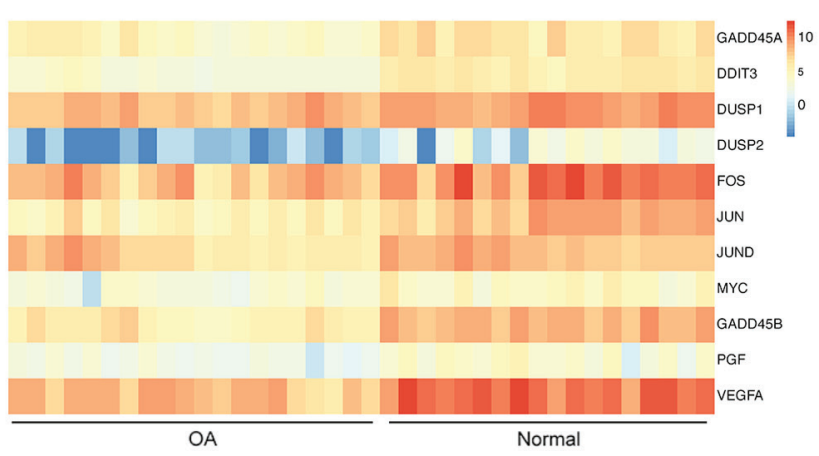

FoxO signaling pathway

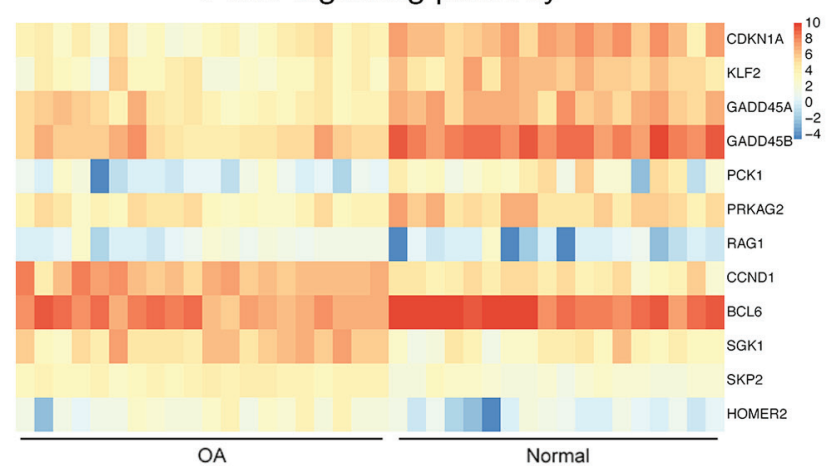

PI3K-Akt signaling pathway

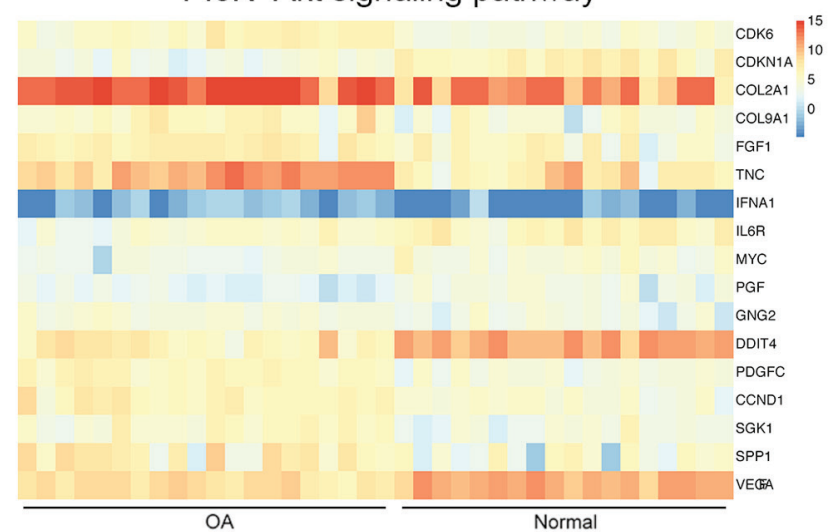

Figure 6. Identification of pivotal signaling pathways. (A) Protein-protein interaction network of key TFs and their target DEGs involved in the pivotal signaling pathways. The node size indicates the degree of interaction. (B) Heatmap of key TFs and their target DEGs involved in the pivotal signaling pathways. TF, transcription factor; DEG, differentially expressed gene; Fox, forkhead box; IL, interleukin; MAPK, mitogen-activated protein kinase; OA, osteoarthritis .

PTPRC, RPF2. GADD45A and GADD45B, members of the GADD45 family, have been implicated in numerous basic processes, and have been demonstrated to be associated with aging and age-associated diseases (54). GADD45A and GADD45B have been reported to interact with AP-1, ATF3 and MYC, which have been identified as key TFs in the present study (55). However, to the best of our knowledge, the roles of GADD45s in the pathogenesis of OA have not been previously assessed, and therefore, further studies are required in order to investigate this. Previous studies have demonstrated that CDC42 is closely associated with the chondrocyte phenotype (56) and age-associated OA (57). A previous study has also indicated that $\mathrm{C} / \mathrm{EBP} \beta$ represses the transcriptional activity of Col2A1 directly and indirectly in ATDC5 cells (58). However, there is currently no evidence that $\mathrm{C} / \mathrm{EBP} \gamma$ directly correlates with OA. 
REL, also termed C-Rel, is a subunit of NF- $\kappa \mathrm{B}$, and it is well established that NF- $\kappa \mathrm{B}$ serves a key role in OA (59). Morrovati et al (60) demonstrated that treatment of chondrocytes with IL-1 $\beta$ resulted in a significant increase in the expression level of C-Rel. However, there is still uncertainty as to whether C-Rel may serve as a therapeutic target for OA. Bradley et al (61) reported that Phlpp1 controls chondrogenesis via multiple mechanisms and that Phlpp1 inhibition may be a strategy to promote cartilage regeneration and repair. However, no previous study has investigated other genes, including NFIL3, MYEF2, IRF4, PTPRC and $\mathrm{RPF} 2$, in articular cartilage, and the results of the present study suggest that they deserve further investigation. The degradation and loss of articular cartilage is a central feature in OA (29). Therefore, the present study utilized the GSE114007 dataset, in which samples were obtained from cartilage tissue. Subchondral bone has received particular attention in recent years (62). In one study, WGCNA, the same method as that used in the present study, was applied to analyze the dataset GSE51588, whose samples were obtained from subchondral bone. They identified another set of core genes, including COL6A3, COL6A1, ITGA11, BAMBI and HCK, to be associated with the pathological processes of subchondral bone in OA (63). However, these results are inconsistent with those obtained by the present study, which indicated that different pathological mechanisms may exist between cartilage tissue and subchondral bone in OA. As summarized by Loeser et al (62), it is indicated that each event (osteophyte formation, subchondral bone sclerosis and cartilage degeneration) is regulated by different mediators.

In addition to the key TFs and hub DEGs discussed above, the present study also identified 4 pivotal signaling pathways in the pathogenesis of OA, including osteoclast differentiation, FoxO, MAPK and the PI3K-Akt signaling pathway. It was also identified that GADD45A and GADD45B participated in the MAPK and FoxO signaling pathways, and that the PI3K/Akt and MAPK signaling pathways are closely associated with MYC and VEGFA. Furthermore, FOS, JUN and JUND were indicated to be involved in osteoclast differentiation and MAPK signaling pathways. It is now well established that in OA, the balance between osteoclast-induced bone resorption and osteoblast-induced remodeling is being progressively deregulated (34). A study by Durand et al (64), by comparing the osteoclastogenic capacity of peripheral blood mononuclear cells (PBMCs) from patients with OA to that of PBMCs from normal individuals, revealed that monocytes from patients with OA have increased osteoclastogenesis and bone resorption. In fact, numerous studies on OA have suggested that enhanced osteoclast-mediated bone resorption is a potential pathological mechanism for OA $(65,66)$. The FoxO signaling pathway has been reported to be a regulator of the pathological processes of OA (67) and the function is perhaps realized by autophagy $(68,69)$. A recent study revealed that FoxO as essential TFs regulating postnatal articular cartilage growth and homeostasis (68). Activation of the MAPK signaling pathway increases the expression of MMPs in chondrocytes (70). In addition, the MAPK signaling pathway is involved in the apoptosis and expression of pro-inflammatory cytokines in human OA chondrocytes (71). Therefore, MAPK signaling is closely associated with the pathological changes of OA, and is a potential therapeutic target for OA. The PI3K signaling pathway is paramount for cell growth and survival (72), with a previous study revealing that the influence of the PI3K/Akt signaling pathway on OA is realized by autophagy (73). The PI3K/Akt pathway may be suppressed by elevated levels of ROS in OA chondrocytes so that inflammatory processes are promoted (74). Furthermore, oxidative stress may cause chondrocyte senescence by activating MAPK and PI3K/Akt signaling pathways. In general, there is a close association between abnormal regulation of pivotal signaling pathways and the disequilibrium of cellular homeostasis and cartilage degeneration.

In summary, the present study identified the hub genes and 5 hub modules associated with OA based on WGCNA. In addition, the present study was the first to identify key TFs through co-expressed gene modules. Although there have been a multitude of academic theses on the pathogenic mechanism of OA, the pathogenesis of OA has remained to be completely elucidated. Novel potential targets and 4 pivotal signaling pathways for therapeutic intervention. The present study provides an important opportunity to advance the understanding of OA. However, the present results are based on a Bioinformatics analysis and further validation is required in order to verify the potential key roles of these genes in the development of OA in vitro and in vivo.

\section{Acknowledgements}

Not applicable.

\section{Funding}

No funding was received.

\section{Availability of data and materials}

The datasets used and/or analyzed during the current study are available from the corresponding author on reasonable request.

\section{Authors' contributions}

XL conceived and designed the study. XG performed data analyses and wrote the manuscript. YS contributed to data acquisition and manuscript revision.

\section{Ethics approval and consent to participate}

Not applicable.

\section{Patient consent for publication}

Not applicable.

\section{Competing interests}

The authors declare that they have no competing interests. 


\section{References}

1. Thomas E, Peat G and Croft P: Defining and mapping the person with osteoarthritis for population studies and public health. Rheumatology (Oxford) 53: 338-345, 2014.

2. Xie F, Kovic B, Jin X, He X, Wang M and Silvestre C: Economic and humanistic burden of osteoarthritis: A systematic review of large sample studies. Pharmacoeconomics 34: 1087-1100, 2016.

3. Goldring MB and Otero M: Inflammation in osteoarthritis. Curr Opin Rheumatol 23: 471-478, 2011.

4. Sellam J and Berenbaum F: The role of synovitis in pathophysiology and clinical symptoms of osteoarthritis. Nat Rev Rheumatol 6: 625-635, 2010 .

5. Tonge DP, Pearson MJ and Jones SW: The hallmarks of osteoarthritis and the potential to develop personalised disease-modifying pharmacological therapeutics. Osteoarthritis Cartilage 22: 609-621, 2014.

6. Hunter DJ and Bierma-Zeinstra S: Osteoarthritis. Lancet 393 $1745-1759,2019$

7. Ren YM, Zhao X, Yang T, Duan YH, Sun YB, Zhao WJ and Tian MQ: Exploring the key genes and pathways of osteoarthritis in knee cartilage in a rat model using gene expression profiling. Yonsei Med J 59: 760-768, 2018.

8. Zhu N, Hou J, Wu Y, Li G, Liu J, Ma G, Chen B and Song Y: Identification of key genes in rheumatoid arthritis and osteoarthritis based on bioinformatics analysis. Medicine (Baltimore) 97: e10997, 2018

9. Langfelder $\mathrm{P}$ and Horvath S: WGCNA: An R package for weighted correlation network analysis. BMC bioinformatics 9 : $559,2008$.

10. Fei Q, Lin J, Meng H, Wang B, Yang Y, Wang Q, Su N, Li J and Li D: Identification of upstream regulators for synovia expression signature genes in osteoarthritis. Joint Bone Spine 83 : 545-551, 2016

11. Fisch KM, Gamini R, Alvarez-Garcia O, Akagi R, Saito M, Muramatsu Y, Sasho T, Koziol JA, Su AI and Lotz MK: Identification of transcription factors responsible for dysregulated networks in human osteoarthritis cartilage by global gene expression analysis. Osteoarthritis Cartilage 26: 1531-1538, 2018

12. Barrett T, Troup DB, Wilhite SE, Ledoux P, Evangelista C, Kim IF, Tomashevsky M, Marshall KA, Phillippy KH, Sherman PM, et al: NCBI GEO: Archive for functional genomics data sets-10 years on. Nucleic Acids Res 39 (Database Issue): D1005-D1010, 2011

13. Ritchie ME, Phipson B, Wu D, Hu Y, Law CW, Shi W and Smyth GK: limma powers differential expression analyses for RNA-sequencing and microarray studies. Nucleic Acids Res 43 e47, 2015.

14. Xin J, Mark A, Afrasiabi C, Tsueng G, Juchler M, Gopal N, Stupp GS, Putman TE, Ainscough BJ, Griffith OL, et al: High-performance web services for querying gene and variant annotation. Genome Biol 17: 91, 2016.

15. Benjamini Y, Drai D, Elmer G, Kafkafi N and Golani I: Controlling the false discovery rate in behavior genetics research. Behav Brain Res 125: 279-284, 2001.

16. Chin CH, Chen SH, Wu HH, Ho CW, Ko MT and Lin CY: cytoHubba: Identifying hub objects and sub-networks from complex interactome. BMC Syst Biol 8 (Suppl 4): S11, 2014.

17. Yu G, Wang LG, Han Y and He QY: clusterProfiler: An R package for comparing biological themes among gene clusters. OMICS 16: 284-287, 2012.

18. Potier D, Atak ZK, Sanchez MN, Herrmann C and Aerts S: Using cisTargetX to predict transcriptional targets and networks in Drosophila. Methods Mol Biol 786: 291-314, 2012.

19. Aerts S, Quan XJ, Claeys A, Naval Sanchez M, Tate P, Yan J and Hassan BA: Robust target gene discovery through transcriptome perturbations and genome-wide enhancer predictions in Drosophila uncovers a regulatory basis for sensory specification. PLoS Biol 8: e1000435, 2010.

20. Ringnér M: What is principal component analysis? Nat Biotechnol 26: 303-304, 2008

21. O'Connor MI and Hooten EG: Breakout session: Gender disparities in knee osteoarthritis and TKA. Clin Orthop Relat Res 469: 1883-1885, 2011.

22. Lee TI and Young RA: Transcriptional regulation and its misregulation in disease. Cell 152: 1237-1251, 2013.

23. Abusarah J, Benabdoune H, Shi Q, Lussier B, Martel-Pelletier J, Malo M, Fernandes JC, de Souza FP, Fahmi H and Benderdour M: Elucidating the role of protandim and 6-Gingerol in protection against osteoarthritis. J Cell Biochem 118: 1003-1013, 2017.
24. Dunn SL, Soul J, Anand S, Schwartz JM, Boot-Handford RP and Hardingham TE: Gene expression changes in damaged osteoarthritic cartilage identify a signature of non-chondrogenic and mechanical responses. Osteoarthritis Cartilage 24: 1431-1440, 2016.

25. Fu M, Huang G, Zhang Z, Liu J, Zhang Z, Huang Z, Yu B and Meng F: Expression profile of long noncoding RNAs in cartilage from knee osteoarthritis patients. Osteoarthritis Cartilage 23: 423-432, 2015

26. Stuart JM, Segal E, Koller D and Kim SK: A gene-coexpression network for global discovery of conserved genetic modules. Science 302: 249-255, 2003.

27. Fujii W, Kawahito Y, Nagahara H, Kukida Y, Seno T, Yamamoto A, Kohno M, Oda R, Taniguchi D, Fujiwara H, et al: Monocarboxylate transporter 4, associated with the acidification of synovial fluid, is a novel therapeutic target for inflammatory arthritis. Arthritis Rheumatol 67: 2888-2896, 2015.

28. Zhu X, Yang S, Lin W, Wang L, Ying J, Ding Y and Chen X: Roles of cell cyle regulators cyclin D1, CDK4, and p53 in knee osteoarthritis. Genet Test Mol Biomarkers 20: 529-534, 2016.

29. Troeberg L and Nagase H: Proteases involved in cartilage matrix degradation in osteoarthritis. Biochim Biophys Acta 1824: 133-145, 2012.

30. Ahmed U, Thornalley PJ and Rabbani N: Possible role of methylglyoxal and glyoxalase in arthritis. Biochem Soc Trans 42: 538-542, 2014.

31. Luo H, Yao L, Zhang Y and Li R: Liquid chromatography-mass spectrometry-based quantitative proteomics analysis reveals chondroprotective effects of astragaloside IV in interleukin-1 $\beta$-induced SW1353 chondrocyte-like cells. Biomed Pharmacother 91: 796-802, 2017.

32. Andriacchi TP, Favre J, Erhart-Hledik JC and Chu CR: A systems view of risk factors for knee osteoarthritis reveals insights into the pathogenesis of the disease. Ann Biomed Eng 43: 376-387, 2015.

33. Scanzello CR, Plaas A and Crow MK: Innate immune system activation in osteoarthritis: Is osteoarthritis a chronic wound? Curr Opin Rheumatol 20: 565-572, 2008.

34. Marchev AS, Dimitrova PA, Burns AJ, Kostov RV, Dinkova-Kostova AT and Georgiev MI: Oxidative stress and chronic inflammation in osteoarthritis: Can NRF2 counteract these partners in crime? Ann N Y Acad Sci 1401: 114-135, 2017.

35. Hess J, Angel P and Schorpp-Kistner M: AP-1 subunits: Quarrel and harmony among siblings. J Cell Sci 117: 5965-5973, 2004.

36. Ionescu AM, Schwarz EM, Vinson C, Puzas JE, Rosier R, Reynolds PR and O'Keefe RJ: PTHrP modulates chondrocyte differentiation through AP-1 and CREB signaling. J Biol Chem 276: 11639-11647, 2001.

37. Schmucker AC, Wright JB, Cole MD and Brinckerhoff CE: Distal interleukin-1 $\beta$ (IL-1 $\beta$ ) response element of human matrix metalloproteinase-13 (MMP-13) binds activator protein 1 (AP-1) transcription factors and regulates gene expression. J Biol Chem 287: 1189-1197, 2012

38. Moritani NH, Kubota S, Eguchi T, Fukunaga T, Yamashiro T, Takano-Yamamoto T, Tahara H, Ohyama K, Sugahara T and Takigawa M: Interaction of AP-1 and the ctgf gene: A possible driver of chondrocyte hypertrophy in growth cartilage. J Bone Miner Metab 21: 205-210, 2003.

39. Papachristou D, Pirttiniemi P, Kantomaa T, Agnantis N and Basdra EK: Fos- and Jun-related transcription factors are involved in the signal transduction pathway of mechanical loading in condylar chondrocytes. Eur J Orthod 28: 20-26, 2006.

40. Kim MG, Yang JH, Kim KM, Jang CH, Jung JY, Cho IJ, Shin SM and Ki SH: Regulation of Toll-like receptor-mediated Sestrin2 induction by AP-1, Nrf2, and the ubiquitin-proteasome system in macrophages. Toxicol Sci 144: 425-435, 2015.

41. Briso EM, Guinea-Viniegra J, Bakiri L, Rogon Z, Petzelbauer P, Eils R, Wolf R, Rincón M, Angel P and Wagner EF: Inflammation-mediated skin tumorigenesis induced by epidermal c-Fos. Genes Dev 27: 1959-1973, 2013.

42. Wu YH, Liu W, Zhang L, Liu XY, Wang Y, Xue B, Liu B, Duan R, Zhang B and Ji Y: Effects of microRNA-24 targeting $\mathrm{C}$-myc on apoptosis, proliferation, and cytokine expressions in chondrocytes of rats with osteoarthritis via MAPK signaling pathway. J Cell Biochem 119: 7944-7958, 2018.

43. Lemos DR, McMurdo M, Karaca G, Wilflingseder J, Leaf IA, Gupta N, Miyoshi T, Susa K, Johnson BG, Soliman K, et al: Interleukin-1 $\beta$ activates a MYC-dependent metabolic switch in kidney stromal cells necessary for progressive tubulointerstitial fibrosis. J Am Soc Nephrol 29: 1690-1705, 2018. 
44. Anderton B, Camarda R, Balakrishnan S, Balakrishnan A, Kohnz RA, Lim L, Evason KJ, Momcilovic O, Kruttwig K, Huang Q, et al: MYC-driven inhibition of the glutamate-cysteine ligase promotes glutathione depletion in liver cancer. EMBO Rep 18: 569-585, 2017.

45. Chen D, Xiong XQ, Zang YH, Tong Y, Zhou B, Chen Q, Li YH, Gao XY, Kang YM and Zhu GQ: BCL6 attenuates renal inflammation via negative regulation of NLRP3 transcription. Cell Death Dis 8: e3156, 2017.

46. Iezaki T, Ozaki K, Fukasawa K, Inoue M, Kitajima S, Muneta T, Takeda S, Fujita H, Onishi Y, Horie T, et al: ATF3 deficiency in chondrocytes alleviates osteoarthritis development. J Pathol 239: 426-437, 2016

47. Chen HH, Lai PF, Lan YF, Cheng CF, Zhong WB, Lin YF, Chen TW and Lin H: Exosomal ATF3 RNA attenuates pro-inflammatory gene MCP-1 transcription in renal ischemia-reperfusion. J Cell Physiol 229: 1202-1211, 2014.

48. Pernhorst K, Herms S, Hoffmann P, Cichon S, Schulz H, Sander T, Schoch S, Becker AJ and Grote A: TLR4, ATF-3 and IL8 inflammation mediator expression correlates with seizure frequency in human epileptic brain tissue. Seizure 22: 675-678, 2013.

49. Walsh DA, McWilliams DF, Turley MJ, Dixon MR, Fransès RE, Mapp PI and Wilson D: Angiogenesis and nerve growth factor at the osteochondral junction in rheumatoid arthritis and osteoarthritis. Rheumatology (Oxford) 49: 1852-1861, 2010.

50. Tan Z, Chen K, Wu W, Zhou Y, Zhu J, Wu G, Cao L, Zhang X, Guan H, Yang Y, et al: Overexpression of HOXC10 promotes angiogenesis in human glioma via interaction with PRMT5 and upregulation of VEGFA expression. Theranostics 8: 5143-5158, 2018.

51. Zan PF, Yao J, Wu Z, Yang Y, Hu S and Li GD: Cyclin D1 gene silencing promotes IL-1 $\beta$-induced apoptosis in rat chondrocytes. J Cell Biochem 119: 290-299, 2018.

52. Lefebvre V and Dvir-Ginzberg M: SOX9 and the many facets of its regulation in the chondrocyte lineage. Connect Tissue Res 58: 2-14, 2017.

53. Li Z, Wang Q, Chen G, Li X, Yang Q, Du Z, Ren M, Song Y and Zhang G: Integration of gene expression profile data to screen and verify hub genes involved in osteoarthritis. Biomed Res Int 2018: 9482726, 2018.

54. Moskalev AA, Smit-McBride Z, Shaposhnikov MV, Plyusnina EN, Zhavoronkov A, Budovsky A, Tacutu R and Fraifeld VE: Gadd45 proteins: Relevance to aging, longevity and age-related pathologies. Ageing Res Rev 11: 51-66, 2012.

55. Amente S, Zhang J, Lavadera ML, Lania L, Avvedimento EV and Majello B: Myc and PI3K/AKT signaling cooperatively repress FOXO3a-dependent PUMA and GADD45a gene expression. Nucleic Acids Res 39: 9498-9507, 2011.

56. Novakofski KD, Torre CJ and Fortier LA: Interleukin-1 $\alpha,-6$, and -8 decrease Cdc 42 activity resulting in loss of articular chondrocyte phenotype. J Orthop Res 30: 246-251, 2012.

57. Fortier LA and Miller BJ: Signaling through the small G-protein Cdc42 is involved in insulin-like growth factor-I resistance in aging articular chondrocytes. J Orthop Res 24: 1765-1772, 2006.

58. Ushijima T, Okazaki $\mathrm{K}$, Tsushima $\mathrm{H}$ and Iwamoto $\mathrm{Y}$ : CCAAT/enhancer-binding protein $\beta$ regulates the repression of type II collagen expression during the differentiation from proliferative to hypertrophic chondrocytes. J Biol Chem 289: 2852-2863, 2014

59. Lepetsos P, Papavassiliou KA and Papavassiliou AG: Redox and $\mathrm{NF}-\kappa \mathrm{B}$ signaling in osteoarthritis. Free Radic Biol Med 132: 90-100, 2019

60. Morrovati F, Karimian Fathi N, Soleimani Rad J and Montaseri A Mummy prevents IL-1 $\beta$-induced inflammatory responses and cartilage matrix degradation via inhibition of NF- $\mathrm{KB}$ subunits gene expression in pellet culture system. Adv Pharm Bull 8: 283-289, 2018
61. Bradley EW, Carpio LR, Newton AC and Westendorf JJ: Deletion of the PH-domain and leucine-rich repeat protein phosphatase 1 (Phlpp1) increases fibroblast growth factor (Fgf) 18 expression and promotes chondrocyte proliferation. J Biol Chem 290: 16272-16280, 2015.

62. Loeser RF, Goldring SR, Scanzello CR and Goldring MB: Osteoarthritis: A disease of the joint as an organ. Arthritis Rheum 64: 1697-1707, 2012.

63. Guo SM, Wang JX, Li J, Xu FY, Wei Q, Wang HM, Huang HQ, Zheng SL, Xie YJ and Zhang C: Identification of gene expression profiles and key genes in subchondral bone of osteoarthritis using weighted gene coexpression network analysis. J Cell Biochem 119: 7687-7695, 2018.

64. Durand M, Komarova SV, Bhargava A, Trebec-Reynolds DP, Li K, Fiorino C, Maria O, Nabavi N, Manolson MF, Harrison RE, et al: Monocytes from patients with osteoarthritis display increased osteoclastogenesis and bone resorption: The in vitro osteoclast differentiation in arthritis study. Arthritis Rheum 65: 148-158, 2013.

65. Bertuglia A, Lacourt M, Girard C, Beauchamp G, Richard H and Laverty S: Osteoclasts are recruited to the subchondral bone in naturally occurring post-traumatic equine carpal osteoarthritis and may contribute to cartilage degradation. Osteoarthritis Cartilage 24: 555-566, 2016.

66. Botter SM, van Osch GJ, Waarsing JH, van der Linden JC, Verhaar JA, Pols HA, van Leeuwen JP and Weinans H: Cartilage damage pattern in relation to subchondral plate thickness in a collagenase-induced model of osteoarthritis. Osteoarthritis Cartilage 16: 506-514, 2008.

67. Akasaki Y, Hasegawa A, Saito M, Asahara H, Iwamoto Y and Lotz MK: Dysregulated FOXO transcription factors in articular cartilage in aging and osteoarthritis. Osteoarthritis Cartilage 22: $162-170,2014$.

68. Matsuzaki T, Alvarez-Garcia O, Mokuda S, Nagira K, Olmer M, Gamini R, Miyata K, Akasaki Y, Su AI, Asahara H and Lotz MK: FoxO transcription factors modulate autophagy and proteoglycan 4 in cartilage homeostasis and osteoarthritis. Sci Transl Med 10: pii: eaan0746, 2018.

69. Shen C, Cai GQ, Peng JP and Chen XD: Autophagy protects chondrocytes from glucocorticoids-induced apoptosis via ROS/Akt/FOXO3 signaling. Osteoarthritis Cartilage 23: 2279-2287, 2015.

70. Zeng GQ, Chen AB, Li W, Song JH and Gao CY: High MMP-1, MMP-2, and MMP-9 protein levels in osteoarthritis. Genet Mol Res 14: 14811-14822, 2015

71. Sun HY, Hu KZ and Yin ZS: Inhibition of the p38-MAPK signaling pathway suppresses the apoptosis and expression of proinflammatory cytokines in human osteoarthritis chondrocytes. Cytokine 90: 135-143, 2017.

72. Chang L, Graham PH and Ni J: Targeting PI3K/Akt/mTOR signaling pathway in the treatment of prostate cancer radioresistance. Crit Rev Oncol Hematol 96: 507-517, 2015.

73. Xue JF, Shi ZM,Zou J and Li XL: Inhibition of PI3K/AKT/mTOR signaling pathway promotes autophagy of articular chondrocytes and attenuates inflammatory response in rats with osteoarthritis. Biomed Pharmacother 89: 1252-1261, 2017.

74. Yu SM and Kim SJ: The thymoquinone-induced production of reactive oxygen species promotes dedifferentiation through the ERK pathway and inflammation through the p38 and PI3K pathways in rabbit articular chondrocytes. Int J Mol Med 35: 325-332, 2015.

This work is licensed under a Creative Commons Attribution-NonCommercial-NoDerivatives 4.0 International (CC BY-NC-ND 4.0) License. 